\title{
Utilización de marcas electrónicas "PIT tags" en Liolaemus y descripción de una técnica de implante para especies de pequeña y mediana talla
}

\section{Use of passive integrated transponder "PIT tags" in Liolaemus from chile and description of a tagging technique for small and medium size lizards}

\author{
Gabriel Lobos*, Constanza Méndez \& Alejandra Alzamora \\ Centro de Estudios de Vida Silvestre (CEVIS), Facultad de Ciencias Veterinarias y Pecuarias, Universidad de Chile, Avenida \\ Santa Rosa 11735, La Pintana, Santiago, Chile. \\ *Email: galobos@ug.uchile.cl
}

\begin{abstract}
RESUMEN
Los estudios de campo con reptiles, requieren en muchas oportunidades de poder contar con métodos de marcaje que permitan el seguimiento de los animales en el mediano y largo plazo y que ellos sean válidos desde la perspectiva bioética. En este trabajo, validamos la utilización de marcas electrónicas "PIT tags" (Passive Integrated Transponder) para el género Liolaemus en Chile. Nuestros resultados muestran que el uso de estas marcas fue satisfactorio en al menos 6 especies de saurios, registrándose recapturas variables según especies y un incremento importante del peso en aquellos animales recapturados. Esta técnica, representa un método de marcaje de baja invasividad en relación a otros métodos clásicos como las mutilaciones.
\end{abstract}

PalABRAs claves: PIT tags, Liolaemus, Chile, marcaje-recaptura, microchips.

\begin{abstract}
Reptile's field studies frequently require counting with tagging methods that allow surveillance procedures of animals both in the medium and long term, and also that they will be valid from a bioethical point of view. In this work we validate the use of electronic tags "PIT tags" (Passive Integrated Transponder) for the Liolaemus genus in Chile. Our results show that the use of these tags was successful in at least 6 lizard species, recording variable recapture -by weight and weight increasein those animals recaptured. This technique represents a low invasiveness method compared to other classic methods such as mutilations.
\end{abstract}

KEYwords: PIT tags, Liolaemus, Chile, mark-recapture, microchips

\section{INTRODUCCIÓN}

El creciente interés por desarrollar estudios de campo (investigación científica, estudios en el marco del Sistema de Evaluación de Impacto Ambiental, monitoreos) en la fauna herpetológica del país, impone el desafío de aplicar metodologías adecuadas para las investigaciones de mediano y largo plazo y que ellas se rijan por principios éticos referidos al bienestar de los animales en estudio. En este contexto, la biología de la conservación aparece como una disciplina pionera en el desarrollo de este tipo de enfoques (Sutherland 2000), planteando la necesidad de aplicar metodologías de investigación éticamente aceptables, donde se incluye el refinamiento de las actuales técnicas de estudio, de acuerdo a los principios planteados por Rusell \& Burch (1959).

La noción de que sólo los vertebrados superiores eran capaces de experimentar dolor, motivó pocos cuestionamientos a las investigaciones en vertebrados como los reptiles. En la actualidad, este enfoque no es aceptado (Ehrensing et al. 1982; Sneddon et al. 2003) y ello ha motivado a las revistas científicas a no publicar trabajos en que vertebrados hayan sido sujeto de prácticas cuestionables no aprobadas por comités de bioética.

En Chile, las nuevas investigaciones comienzan a recoger estos planteamientos. Los viejos métodos de marcaje de 
herpetozoos mediante amputaciones (ortejos y dedos, en el caso de anfibios y reptiles) están cuestionados (al menos por el organismo estatal que rige a la fauna silvestre del país, Servicio Agrícola y Ganadero de Chile) y comienzan a ser reemplazados por métodos menos invasivos. Del mismo modo, resulta obligatorio que los proyectos financiados por CONICYT (Comisión Nacional de Investigación Científica y Tecnológica), la principal organización financiadora de proyectos de investigación, deban someterse a los respectivos Comités de las facultades o institutos a los que ellos pertenecen. Adicionalmente, a partir del año 2005 se creó una Comisión Asesora de Ética y Bioética de FONDECYT (Fondo Nacional de Desarrollo Científico y Tecnológico) (Santos 2005).

En este contexto, el uso de marcas electrónicas "PIT tags" (Passive Integrated Transponder) es hoy una tecnología de un costo accesible y adecuada para animales como los reptiles, en que clásicamente se han utilizados pinturas de corta duración (dado el cambio de piel que estos animales experimentan) y mutilaciones. Otra técnica utilizada en reptiles han sido las mostacillas de colores que se suturan a la base de la cola (Araya 2007; Frutos et al. 2007), las que si bien son de baja invasividad, son más limitadas en cuanto al número de combinaciones y riesgo de pérdida de las marcas.

Un problema que surge con el uso de "PIT tags" en saurios chilenos, dice relación con la pequeña talla de gran parte de los representantes nacionales (Vidal 2008), donde predominan las lagartijas (especies con un largo hocico cloaca (LHC) menor a $7 \mathrm{~cm}$ ) por sobre los lagartos de mayor talla (LHC mayor a $7 \mathrm{~cm}$ ), de acuerdo a Nuñez y Jaksic (1992). Si bien se recomienda que los dispositivos se utilicen en proporciones de que su peso no supere al 2 a $3 \%$ del peso del animal (Grubert 2004), en la práctica, animales de talla media (para nosotros aquellos con LHC entre 6 y $7 \mathrm{~cm}$ ) y pequeña (para nosotros HLC menor a $6 \mathrm{~cm}$ ), son complejos de marcar al considerar el tamaño y calibre del trocar de la jeringa de implante $(31,7 \mathrm{~mm}$. de largo y $4 \mathrm{~mm}$. de ancho). Lo anterior, probablemente ha sido parte de las motivaciones para el escaso uso de esta técnica en lagartos chilenos. No obstante, los "PIT tags" son ampliamente utilizados en estudios de anfibios (Brown 1997; Johnson \& Wallace 2002; Pope \& Matthews 2001) y reptiles (Boarman et al. 1998; Elbin \& Burger 1994; Germano \& Williams 1993; Jemison et al. 1995; Keck 1994).

En este trabajo reportamos antecedentes respecto al uso de PIT tags en representantes del género Liolaemus en Chile y describimos una técnica para su aplicación en animales de talla pequeña a mediana, con el objetivo de contribuir al desarrollo de los estudios de campo que requieren de técnicas de marcaje y recaptura. Por otro lado, se cuantificó el peso de los individuos, con el fin de determinar efectos negativos de los PIT tags sobre éstos.

\section{MATERIALES Y MÉTODOS}

\section{ESPECIES OBJETIVO}

Durante los años 2006 al 2009 realizamos estudios de marcaje y recaptura en representantes del género Liolaemus en varias regiones de Chile, como parte de monitoreos ejecutados por los autores dentro del Sistema de Evaluación de Impacto Ambiental. Las capturas se concentraron en las estaciones de primavera-verano, cuando estos animales presentan una mayor actividad biológica (Tabla 1). Para las capturas de los ejemplares, se utilizó la técnica del lazo con nudo escurridizo, técnica de amplio uso en saurios (Sutherland 2000). Se escogieron 6 especies que presentaron el mayor número de animales marcados $\mathrm{y}$ recapturados (366 individuos) y que correspondieron a Liolaemus nitidus (Wiegmann, 1835), L. tenuis (Duméril \& Bibron, 1837), L. monticola (Müller \& Hellmich, 1950), L. pictus (Duméril \& Bibron, 1837), L. platei (Werner, 1898) y L. constanzae (Donoso-Barros, 1961). Otras especies no consideradas en este estudio fueron $L$. fuscus (Boulenger, 1885), L. chiliensis (Lesson, 1831) y L. cyanogaster (Duméril \& Bibron, 1837), donde se marcaron muy pocos ejemplares para su posterior seguimiento. Los animales capturados, fueron mantenidos en bolsas herpetológicas de género y depositados en cajas plásticas de $36 \times 25 \times 25 \mathrm{~cm}$ con sustrato y piedras del mismo lugar de captura. En el terreno, cada individuo fue pesado con pesolas de mano (10 y 30 gr), sexado y marcado. Todo el proceso de toma de datos por animal, no demoro más de 5 minutos, sin embargo, los animales se mantuvieron en observación (cajas de contención) por un lapsus de 1 hora previa a su liberación en los mismos puntos de captura.

Para evaluar un potencial efecto del sexo en la sobrevida de los animales marcados y recapturados, nosotros comparamos la diferencias de pesos por sexo en las especies con mayores recapturas (L. nitidus, L. tenuis y L. constanzae) por medio de la prueba no paramétrica de Wilcoxon (Zar 1996).

Descripción de la téCnICA DE MARCAJE

Para el marcaje de los animales se utilizó dispositivos "PIT tag" de 2,07 mm. de ancho, 12,50 mm. de largo, $125 \mathrm{kHz}$ de frecuencia y 0,1020 gr de peso (Biomark Inc. www.biomark. com). Los dispositivos fueron inyectados con un trocar de calibre de $4 \mathrm{~mm}$. por medio de una inserción quirúrgica en la zona media dorsal del reptil. Los dispositivos se encuentran en un estado pasivo, hasta que son activados por un campo electromagnético generado por el equipo lector (Gibbons \& Andrews 2004). 


\section{PASO 1}

Los saurios son sujetados desde la base de la pata izquierda (para un operador diestro), una vez que el animal se encuentra tranquilo, se prosigue con el procedimiento de marcaje (Fig. 1a).

\section{Paso 2}

Con el extremo filudo del trocar de la jeringa de implante, se realiza una pequeña incisión en la piel del animal, de no más de $3 \mathrm{~mm}$. de ancho, tamaño suficiente para el implante del PIT tag. Se recomienda realizar la incisión en la parte dorsal posterior del animal. Realizada la incisión, se retira suavemente la jeringa y su aguja (Fig. 1b).

\section{Paso 3}

Con la punta de los dedos (pulgar e índice) se toma un PIT tag y se introduce por la incisión realizada, suavemente. Primero se recomienda introducir la punta del PIT tag y posteriormente se introduce por completo (Fig. 1c y d). Una vez introducido el dispositivo, con los dedos se debe direccionar hacia la parte anterior y lateral del animal, ello minimiza la potencial perdida de las marcas. Lo anterior es fácil de ejecutar cuando el dispositivo queda instalado subcutáneamente (Fig. 1e). En algunas ocasiones, si la incisión es profunda, el dispositivo puede alcanzar la cavidad celómica (en especial en animales de talla pequeña), con el riesgo de perforar algún órgano, por lo tanto, es importante que la incisión sea superficial y con el trocar en un ángulo casi horizontal.

\section{Paso 4.}

Con respecto a las medidas de higiene, la figura $1 \mathrm{f}$ muestra los componentes principales del equipo de marcaje, correspondiente a lector, la jeringa de implante y los "PIT tags". El procedimiento debe realizarse con guantes quirúrgicos libres de talco. La superficie de operación en el campo debe ser de un material de fácil limpieza (por ejemplo, la tapa de una caja de plástico) y desinfectada continuamente con alcohol de $70^{\circ}$. Los "PIT tags" a utilizar deben depositarse sobre un algodón con alcohol y el trocar de la jeringa debe ser desinfectado entre cada animal que sea procesado. Adicionalmente, se pude aplicar un adhesivo quirúrgico para asegurar el cierre de los bordes de la herida (a base de cianoacrilatos, disponibles en presentaciones para uso humano y veterinario). Todo el procedimiento debe realizarse en silencio, a objeto de minimizar el estrés sobre los animales. Una vez que se logra práctica, el procedimiento no dura más de 1 minuto, por lo que no se recomienda el uso de agentes anestésicos.

En general, las diferentes especies de lagartos que marcamos, se mantuvieron pasivos durante el procedimiento.

\section{RESULTADOS}

El estudio consideró un total de 366 ejemplares de 6 especies (Tabla 1). Las tasas de recapturas para las especies fluctuaron entre un 12 a un $29 \%$ (Tabla 2). Dentro de los animales marcados, destacan individuos recapturados en períodos que fluctuaron entre 17 días hasta 1 año y 7 meses (en función de la duración de los respectivos monitoreos). En general en estos animales no se observó presencia de lesiones externas evidentes.

Nosotros consideramos al incremento de peso entre el período del marcaje hasta la última captura, como un indicador de la buena condición corporal de los animales en relación al procedimiento de marcaje. De acuerdo a los resultados, en los animales de talla media, un $65 \%$ incrementó su peso ( $13 \%$ lo mantuvo y $22 \%$ disminuyó), mientras que en los de talla pequeña, un $70 \%$ incrementó de peso (10\% lo mantuvo y $20 \%$ disminuyó) (Tabla 3). En relación a un posible efecto del sexo en la sobrevivida de los animales recapturados (condición corporal), la prueba de Wilcoxon no mostró dicho efecto en L. nitidus, L.tenuis y L. constanzae $(\mathrm{W}=8,5 ; \mathrm{p}=0,11, \mathrm{~W}=36 ; \mathrm{p}=0,66, \mathrm{~W}=208$; $\mathrm{p}=0,88$ respectivamente). 

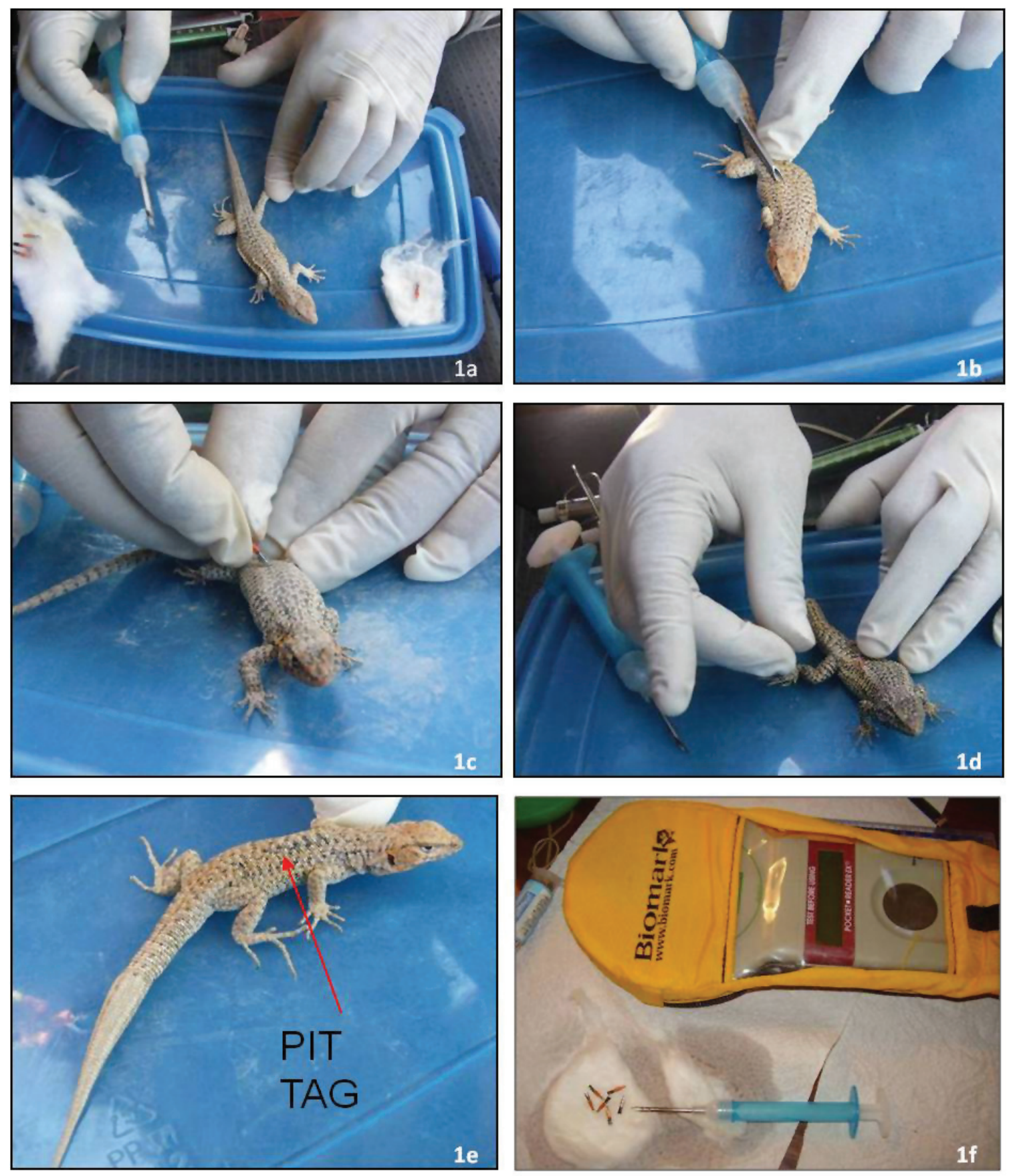

Figura 1. Implante de un "PIT tag" en un Liolaemus constanzae. (a) Sujeción del animal, (b) realización de la incisión, (c-d) implante subcutáneo del dispositivo, (e) animal con dispositivo subcutáneo y (f) componentes principales del equipo de marcaje.

FIGURE 1. "PIT tag" implant in a Liolaemus constanzae specimen. (a) Animal restrains, (b) incision procedures, (c-d) subcutaneous implant of the device, (e) animal carrying subcutaneous device and (f) main components of the tagging equipment. 


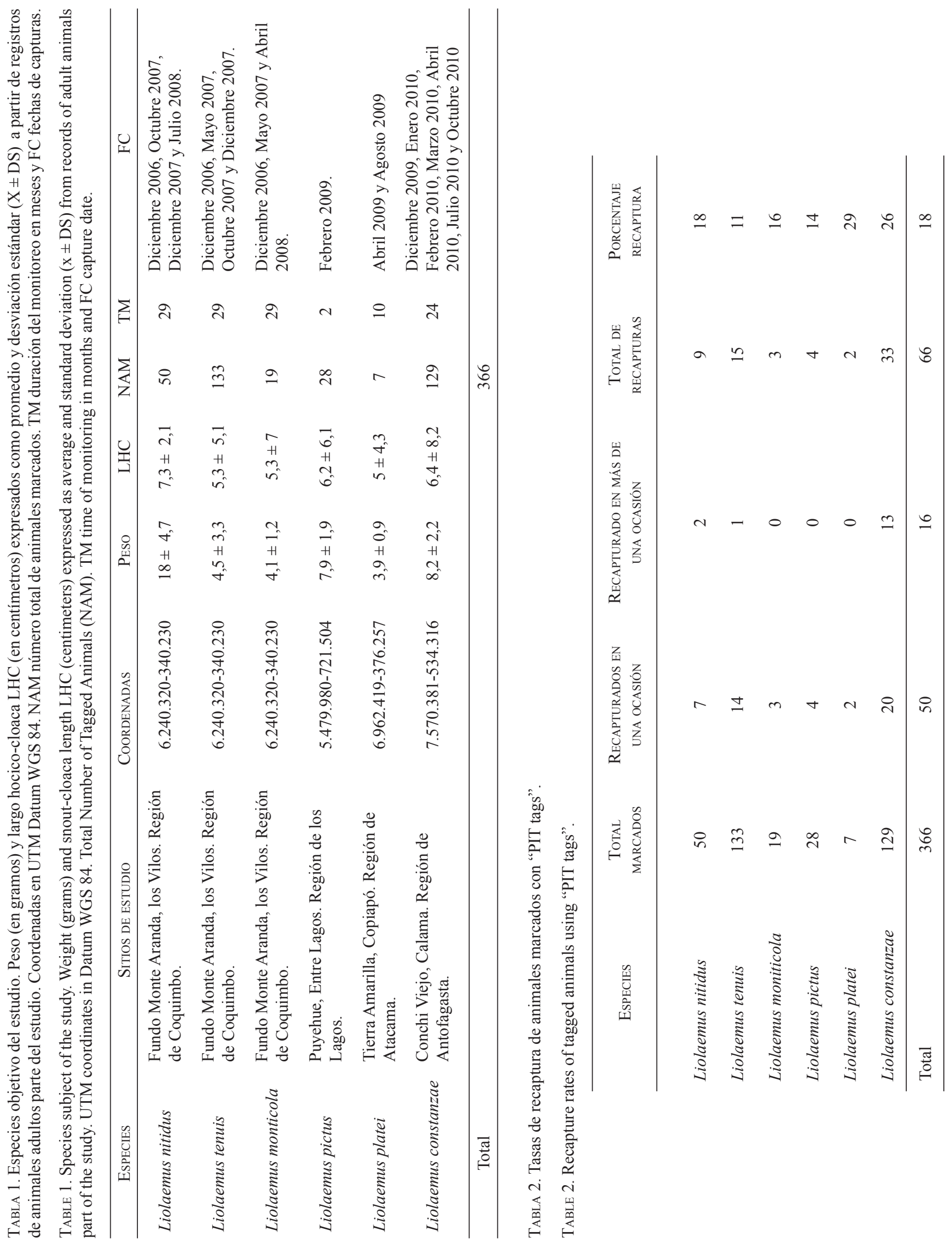


Técnicas de implante en Liolaemus: GABRIEL LoBOS ET AL

Tabla 3. Máximo tiempo de captura (en días) y pesos (en gramo) de animales marcados con "PIT-tags".

TABLE 3. Maximum capture time (days) and weights (grams) of of tagged animals using "PIT tags".

\begin{tabular}{|c|c|c|c|c|}
\hline ESPECIES & $\begin{array}{l}\text { MÁXIMO TIEMPO DE } \\
\text { CAPTURA }\end{array}$ & $\begin{array}{l}\text { PESO INICIAL AL MOMENTO } \\
\text { DEL MARCAJE EN GRAMOS }\end{array}$ & $\begin{array}{c}\text { Peso duRANTE EL } \\
\text { ÚLTIMO EVENTO DE } \\
\text { CAPTURA }\end{array}$ & DIFERENCIA EN $\%$ \\
\hline Liolaemus nitidus & 576 & 15 & 16 & 6,7 \\
\hline Liolaemus nitidus & 240 & 3,5 & 11 & 214,3 \\
\hline Liolaemus nitidus & 30 & 16,5 & 16 & $-3,0$ \\
\hline Liolaemus nitidus & 82 & 6,6 & 15 & 127,3 \\
\hline Liolaemus nitidus & 47 & 11 & 13 & 18,2 \\
\hline Liolaemus nitidus & 17 & 13 & 13 & 0,0 \\
\hline Liolaemus nitidus & 295 & 9,8 & 13 & 32,7 \\
\hline Liolaemus nitidus & 180 & 15 & 20 & 33,3 \\
\hline Liolaemus nitidus & 270 & 18 & 18 & 0,0 \\
\hline Liolaemus tenuis & 313 & 5 & 5,4 & 8,0 \\
\hline Liolaemus tenuis & 19 & 6 & 6 & 0,0 \\
\hline Liolaemus tenuis & 82 & 5,4 & 3,7 & $-31,5$ \\
\hline Liolaemus tenuis & 84 & 4,8 & 4,6 & $-4,2$ \\
\hline Liolaemus tenuis & 365 & 4,8 & 4,5 & $-6,3$ \\
\hline Liolaemus tenuis & 365 & 4,3 & 4,8 & 11,6 \\
\hline Liolaemus tenuis & 365 & 3,4 & 4,1 & 20,6 \\
\hline Liolaemus tenuis & 365 & 3,6 & 4,5 & 25,0 \\
\hline Liolaemus tenuis & 330 & 4,2 & 4,6 & 9,5 \\
\hline Liolaemus tenuis & 420 & 4,8 & 4,5 & $-6,3$ \\
\hline Liolaemus tenuis & 330 & 4,9 & 6,3 & 28,6 \\
\hline Liolaemus tenuis & 365 & 4,4 & 4,6 & 4,5 \\
\hline Liolaemus tenuis & 420 & 4,5 & 4,6 & 2,2 \\
\hline Liolaemus tenuis & 90 & 4,5 & 4,6 & 2,2 \\
\hline Liolaemus tenuis & 90 & 4 & 4,3 & 7,5 \\
\hline Liolaemus montícola & 46 & 4,7 & 5,2 & 10,6 \\
\hline Liolaemus montícola & 180 & 2,7 & 3,5 & 29,6 \\
\hline Liolaemus montícola & 180 & 4,1 & 4,5 & 9,8 \\
\hline Liolaemus pictus & 60 & 9,1 & 9,3 & 2,2 \\
\hline Liolaemus pictus & 60 & 12 & 11 & $-8,3$ \\
\hline Liolaemus pictus & 60 & 5 & 8 & 60,0 \\
\hline Liolaemus pictus & 60 & 11 & 11 & 0,0 \\
\hline Liolaemus platei & 150 & 3 & 3 & 0,0 \\
\hline Liolaemus platei & 150 & 4,5 & 5 & 11,1 \\
\hline
\end{tabular}


Gayana 77(1), 2013

\begin{tabular}{|c|c|c|c|c|}
\hline ESPECIES & $\begin{array}{l}\text { MÁxIMO TIEMPO DE } \\
\text { CAPTURA }\end{array}$ & $\begin{array}{l}\text { PESO INICIAL AL MOMENTO } \\
\text { DEL MARCAJE EN GRAMOS }\end{array}$ & $\begin{array}{c}\text { Peso duRANTE EL } \\
\text { ÚLTIMo EVENTO DE } \\
\text { CAPTURA }\end{array}$ & DIFERENCIA EN \% \\
\hline Liolaemus constanzae & 250 & 6 & 8,5 & 41,7 \\
\hline Liolaemus constanzae & 250 & 5 & 7 & 40,0 \\
\hline Liolaemus constanzae & 90 & 2 & 4,6 & 130,0 \\
\hline Liolaemus constanzae & 163 & 5 & 6 & 20,0 \\
\hline Liolaemus constanzae & 250 & 10 & 10 & 0,0 \\
\hline Liolaemus constanzae & 150 & 6 & 8,5 & 41,7 \\
\hline Liolaemus constanzae & 101 & 9,3 & 10 & 7,5 \\
\hline Liolaemus constanzae & 163 & 4,5 & 5,8 & 28,9 \\
\hline Liolaemus constanzae & 210 & 6 & 8,7 & 45,0 \\
\hline Liolaemus constanzae & 60 & 7,5 & 7,1 & $-5,3$ \\
\hline Liolaemus constanzae & 66 & 2,9 & 4 & 37,9 \\
\hline Liolaemus constanzae & 90 & 6,6 & 7,6 & 15,2 \\
\hline Liolaemus constanzae & 150 & 7,6 & 8,5 & 11,8 \\
\hline Liolaemus constanzae & 45 & 7 & 6 & $-14,3$ \\
\hline Liolaemus constanzae & 39 & 4,9 & 5,1 & 4,1 \\
\hline Liolaemus constanzae & 103 & 5 & 4,7 & $-6,0$ \\
\hline Liolaemus constanzae & 90 & 3,6 & 4,5 & 25,0 \\
\hline Liolaemus constanzae & 131 & 7 & 6 & $-14,3$ \\
\hline Liolaemus constanzae & 163 & 6 & 6,1 & 1,7 \\
\hline Liolaemus constanzae & 60 & 12 & 10,5 & $-12,5$ \\
\hline Liolaemus constanzae & 15 & 5,1 & 5,1 & 0,0 \\
\hline Liolaemus constanzae & 30 & 11 & 10 & $-9,1$ \\
\hline Liolaemus constanzae & 30 & 7,7 & 9 & 16,9 \\
\hline Liolaemus constanzae & 30 & 9,3 & 9 & $-3,2$ \\
\hline Liolaemus constanzae & 60 & 4,1 & 4,5 & 9,8 \\
\hline Liolaemus constanzae & 99 & 4,1 & 5,2 & 26,8 \\
\hline Liolaemus constanzae & 30 & 7 & 7,8 & 11,4 \\
\hline Liolaemus constanzae & 30 & 6,6 & 6,6 & 0,0 \\
\hline Liolaemus constanzae & 240 & 13 & 14 & 7,7 \\
\hline Liolaemus constanzae & 120 & 10 & 12 & 20,0 \\
\hline Liolaemus constanzae & 90 & 11 & 12 & 9,1 \\
\hline Liolaemus constanzae & 150 & 7,5 & 9 & 20,0 \\
\hline Liolaemus constanzae & 90 & 10,3 & 9,8 & $-4,9$ \\
\hline
\end{tabular}




\section{DISCUSIÓN}

En Chile existen escasos antecedentes científicos sobre el marcaje de reptiles y menos de la efectividad de los métodos utilizados. Dentro de los métodos se señalan claves con corte de dedos para estudios de ámbito de hogar de Phymaturus (Habit y Ortiz 1994), combinaciones de cortes de dedos y pinturas para estudio de conducta social en reptiles de la cordillera andina de Chile central (Fox \& Shipman 2003) y mostacillas de colores para estudio de ámbito de hogar en Phymaturus (Araya 2007); por lo general todos estudios de corta duración y no orientados a monitoreos de larga data. En relación al uso de mostacillas, un estudio en Argentina (Halloy \& Robles 2002 para Liolaemus quilmes), monitoreó el ámbito de hogar y fidelidad al sitio durante 3 años consecutivos. Con este método fue posible determinar que en el caso de los machos, 5 animales permanecieron en el sitio durante los 3 años consecutivos (total de 25 machos marcados), al igual que 2 hembras (total 21 hembras marcadas).

De acuerdo a nuestra indagación, no encontramos trabajos con microchips en reptiles chilenos. Los resultados obtenidos muestran que el marcaje con "PIT tags" en representantes de Liolaemus, representa una técnica de baja invasividad apta para su utilización en reptiles de talla mediana y pequeña. Por otra parte, la masificación en el uso de estos dispositivos, han disminuido de modo significativo su costo. Si bien en este estudio no se observó efectos indeseados al usar los denominados "microchips" (como infecciones o evisceraciones), hoy es posible refinar la técnica con la disponibilidad de "Nanochips" más pequeños aún $(1,25$ $\mathrm{mm}$. de ancho y $7 \mathrm{~mm}$. de largo).

En relación a la seguridad de los "PIT tags", no se ha observado que ellos influyan en las tasas de crecimiento, apareamiento, y susceptibilidad a depredadores (Gibbons \& Andrews 2004). En relación a las posibles perdidas de las marcas, ellas solo ocurren por la mala técnica al momento del implante, especialmente al poco tiempo de la inyección, si la apertura ocasionada por la aguja queda abierta, lo que puede ser minimizado con el uso de adhesivos quirúrgicos (Gibbons \& Andrews 2004). Por otra parte, un estudio comparativo entre animales marcados por registros fotográficos y chips, no mostró diferencias de estos últimos sobre las tasas de recaptura y condición corporal (Jehle \& Hold 1998). Las marcas han sido usadas en animales de pequeño peso, como la salamandra Ambystoma opacum de 2 a 4 gr con buenos resultados (Oit \& Scott 1999). Keck 1994, utilizó marcas en serpientes neonatales Thamnophis marcianus de 2,5 gr, sin observar efectos sobre el crecimiento, reportando daño físico solo en 3 de los 12 animales marcados (evisceración por el agujero de inserción del chip en dos animales y rotación de la escama anterior a la zona de inserción del chip en un animal).

En Chile, la aplicación de la legislación ambiental (Ley 19.300 de Bases del Medio Ambiente), ha motivado la implementación de medidas de mitigación, reparación y compensación de impactos ambientales. Dentro de estas medidas, los reptiles han sido un grupo foco de ellas, dada su menor capacidad de movimiento y alto número de especies amenazadas. Lo anterior, ha dado paso a planes de translocación (Craven et al. 1998; Shine \& Koening 2001; Fisher \& Lindenmayer 2000), motivando la necesidad de contar con métodos adecuados de seguimiento en los trabajos de campo. Por ello, resulta necesario poder contar con métodos de marcajes de larga duración y confiabilidad, a objeto de poder evaluar la efectividad de este tipo de medidas.

Finalmente, si bien los "PIT tags" representan una interesante herramienta para el monitoreo de herpetozoos, la aplicación de ellos, debería ser sujeto de un estricto control y apego a protocolos de bioseguridad y de certificación de los profesionales que manipularán animales con fines de marcaje. En este sentido, resultaría necesaria la acreditación de los profesionales autorizados, al igual como se requiere para el anillamiento de aves silvestres, actividad que deberá ser regulada por el Servicio Agrícola y Ganadero de Chile.

\section{AGRADECIMIENTOS}

Los autores expresan su gratitud a dos revisores anónimos, por los valiosos comentarios para el mejoramiento de este escrito.

\section{BIBLOGRAFIA}

Araya, S. 2007. Aspectos autoecológicos relevantes para la conservación de Phymaturus flagellifer (Reptilia, Tropiduridae) en la Reserva Nacional Altos de Lircay, Región del Maule. Memoria para optar al título profesional de Ingeniero en Recursos Naturales Renovables, Facultad de Ciencias Agronómicas, Universidad de Chile. 41 pp.

Boarman, W.I., Beigel, M.L., Goodlett, G.C. \& Sazaki, M. 1998. Passive integrated transponder system for tracking animal movements. Wildlife Society Bulletin 26: 886-891.

Brown, L.J. 1997. Evaluation of some marking and trapping techniques currently used in the study of anuran population dynamics. Journal of Herpetology 31: 410-419.

Craven, S., Barnes, T. \& Kania G. 1998. Toward a professional position on the translocation of problem wildlife. Wildlife Society Bulletin 26:171-177.

Ehrensing, R.H., Michell, G.F. \& Kastin, A.J. 1982. Similar antagonism of morphine analgesia by MIF - 1 and naxolone in Carassius auratus. Pharmacology Biochemistry and Behavior. 17: 757-761. 
Elbin, S.B. \& Burger, J. 1994. Implantable microchips for individual identification in wild and captive populations. Wildlife Society Bulletin 22: 677-683.

Fisher, J. \& Lindermayer, D.B. 2000. An assessment of the published results of animal relocations. Biological Conservation 96: 1-11.

Fox S.F. \& ShIPMAn P.A. 2003. Social behavior at high and low elevations: environmental release and phylogenetic effects in Liolaemus: 310-351. En Fox S.F.; McCoy J.K. \& Baird T.A. (eds). Lizard social behavior. John Hopkins University Press, New York, USA. 438 pp.

Frutos, N., Camporro, L.A. \& Avila, L,J. 2007. Ámbito de hogar de Liolaemus melanops Burmeister, 1888 (Squamata: Liolaemini) en el centro de Chubut, Argentina. Gayana 71 (2): 142-149.

Habit, E.M. \& Ortiz, J.C. 1994. Ámbito de hogar de Phymaturus flagellifer (Reptilia: Tropiduridae). Boletín de la Sociedad de Biología de Concepción 65: 149-152.

Halloy, M. \& Robles, C. 2002. Spatial distribution in a neotropical lizard, Liolaemus quilmes (Liolaemidae): site fidelity and overlapping among males and females. Bulletin of Maryland Herpetological Society 38: 118-129.

Germano, D.J. \& Williams, D.F. 1993. Field evaluation of using Passive Integrated Transponder (PIT) tags to permanently mark lizards. Herpetological Review 24: 54-56.

Gibbons, J.W. \& Andrews, K.M. 2004. PIT Tagging: simple technology at its best. Bioscience 54 (5): 447-454.

Gruber, B. 2004. Measuring activity of geckos with an automatic movement monitoring system. Herpetological Review 35 (3): 245-247.

Jehle, R. \& Hold N. 1998. Pits versus patters: effects of transponders on recapture rate and body condition of Danube crested newst (Triturus dobrogicus) and common spadefoot toads (Pelobates fuscus). Herpetological Journal (8): 181-186.

Jemison, S.C., Bishop, L.A., May, P.G. \& Farell, T.M. 1995. Impact of PIT-tags on growth and movement of the rattlesnake. Journal of Herpetology 29: 129-132.

Johnson, B.R. \& Wallace, J.B. 2002. In situ measurement of larval salamander growth using individuals marked with acrylic polymers. Herpetological Review 33: 29-32.

Keck, M.B. 1994. Test for detrimental effects of PIT tags in neonatal snakes. Copeia 1: 226-228.

NuÑEZ, H. \& JAKSIC F.M. 1992. Lista comentada de los reptiles terrestres de Chile continental. Boletín del Museo Nacional de Historia Natural de Chile 43: 63-91.

Oiт, J. \& ScotT, D.E. 1999. Effects of toe-clipping and PIT- tagging on growth and survival in metamorphic Ambystoma opacum. Journal of herpetology 33: 344-348.

Pope, K.L. \& Matthews, K.R. 2001. Movement ecology and seasonal distribution of mountain yellow-legged frogs in a high-elevation Sierra Nevada Basin. Copeia 2001: 787793.

Rusell, W.M.S. \& Burch, R.L. 1959. The principles of humane experimental technique. London: Methuen. Reprinted by UFAW, 1992: 8 Hamilton Close, South Mimms, Potters Bar, Herts EN6 3 QD England. Society for Assisted Reproductive Technology.

SANTos, M.J. 2005. La Bioética y FONDECYT: 9-20. En: Kottow $\mathrm{M}$ (ed.) Bioética e investigación con seres humanos y en animales. Comité Asesor de Bioética de CONICYT. Comisión Nacional de Investigación Científica y Tecnológica. Santiago, Chile.

Sneddon, L.U., Braithwaite, V.A. \& Gentle, M.J. 2003. Do fishes have nociceptors? Evidence for the evolution of a vertebrate sensory system. Proceeding of the Royal Society of London B (270): 1115-1121.

Shine, R. \& Koening, J. 2001. Snakes in the garden: an analysis of reptiles "rescued" by community based wildlife carers. Biological Conservation 102: 271-283.

Sutherland, W.J. 2000. The conservation handbook: research, management and policy. Blackwell Science, London. 278 p.

VIDAL, M.A. 2008. Biogeografía de anfibios y reptiles. En: Herpetología de Chile (Eds. VIDAL, M. \& LABRA, A.), pp: 195-231. Science Verlag, Chile.

ZAR, J.H. 1996. Biostatistical analysis. Prentice Hall, Upper Saddle River, New Yersey, USA. 660 P.

Recibido: 13.04 .12

Aceptado: 18.10 .12 